\title{
Spotlight on once-monthly long-acting injectable aripiprazole and its potential as maintenance treatment for bipolar I disorder in adult patients
}

This article was published in the following Dove Press journal:

Neuropsychiatric Disease and Treatment

\section{Vanessa Torres-Llenza \\ Pooja Lakshmin \\ Daniel Z Lieberman}

Department of Psychiatry and Behavioral Sciences, George Washington University School of Medicine and Health Sciences, Washington, DC, USA

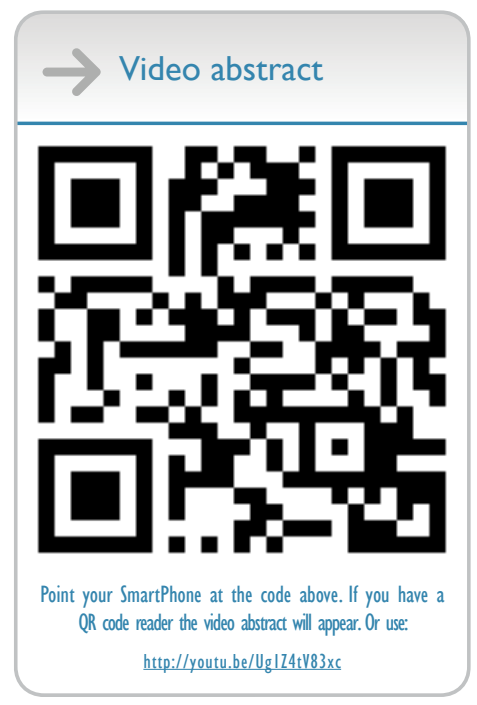

Correspondence: Vanessa Torres-Llenza Department of Psychiatry and Behavioral Sciences, George Washington University School of Medicine and Health Sciences, 2 I 20 L St NW Suite 600, Washington, DC 20037, USA

$\mathrm{Tel}+|20274| 2900$

Email vtorresllenza@mfa.gwu.edu
Abstract: The lack of long-term medication adherence is a challenge in the treatment of bipolar disorder, particularly during the maintenance phase when symptoms are less prominent. The rate of nonadherence is $\sim 20 \%-60 \%$ depending on how strict a definition is used. Nonadherence worsens the course of bipolar disorder and can add hundreds of thousands of dollars to the lifetime cost of treating the illness. Long-acting injectable (LAI) medication is an attractive alternative to daily dosing of oral medication, especially among patients who are ambivalent about treatment. The purpose of this paper is to review the evidence for the safety and efficacy of LAI aripiprazole, which was recently approved for the treatment of bipolar disorder. The approval was based on a single double-blind, placebo-controlled, multisite trial that recruited participants from 103 sites in 7 countries. A total of 731 participants with bipolar disorder were enrolled in the study. Out of that total, 266 were successfully stabilized on LAI aripiprazole and entered the randomization phase. Treatment-emergent adverse events were, for the most part, mild to moderate. Akathisia was the most common adverse event, which, combined with restlessness, was experienced by $23 \%$ of the sample. At the end of the 52 -week study period, nearly twice as many LAI-treated participants remained stable compared to those treated with placebo. Stability during the maintenance phase is arguably the most important goal of treatment. It is during this period of relative freedom from symptoms that patients are able to build a meaningful and satisfying life. The availability of a new treatment agent, particularly one that has the potential to enhance long-term adherence, is a welcome development.

Keywords: antipsychotic, adherence, partial agonist, mood stabilizer, review

\section{Introduction}

Bipolar disorder is an illness of cyclic mood episodes that may be elevated, depressed, or mixed. It affects $2.4 \%$ of the population worldwide. ${ }^{1}$ Episodes of mania or hypomania are characterized by elevated or irritable mood, decreased need for sleep, grandiosity, pressured speech, increased goal-directed activities, high-risk behaviors, distractibility, and flight of ideas. ${ }^{2}$ Depressive episodes are often indistinguishable from symptom presentations in major depressive disorder, placing bipolar patients at risk of misdiagnosis. All aspects of bipolar disorder can be significantly debilitating and interfere with activities of daily living, including interpersonal relationships, and work productivity.

\section{Adherence}

One of the most pressing challenges in the treatment of bipolar disorder is the lack of consistent medication adherence. Adherence has been broadly defined as the extent to 
which a person's behavior coincides with medical advice; ${ }^{3,4}$ however, there is no single way of measuring it. It can be quantified based on how many prescriptions are filled, the degree to which a patient takes medication in the way it was prescribed, or the percentage of prescribed doses that were consumed, for example, $\geq 90 \%$.

In bipolar disorder, nonadherence rates are between $20 \%$ and $60 \% .^{5}$ These rates depend on the definition of nonadherence that is being used, as well as the duration of the study and the characteristics of the patient population. ${ }^{6}$

Sajatovic et al retrospectively assessed adherence to antipsychotic medication among a sample of veterans with bipolar disorder. ${ }^{7}$ They defined adherence using the medication possession ratio (MPR), which is calculated based on how many days' worth of medication a patient actually obtained divided by how many days he/she should have been consuming it. An advantage of using this definition is that the data needed to calculate it can be obtained from the medical chart and pharmacy records, making it unnecessary to interview each patient individually. The disadvantage is that it does not take into account the fact that a patient may have obtained a supply of pills and then failed to take them.

Full adherence in this study was defined as an MPR of $\geq 0.8$. Partial adherence was defined as $\geq 0.5$ to $<0.8$ and patients with an MPR of $<0.5$ were classified as nonadherent. The investigators found that approximately half of bipolar veterans who were prescribed an antipsychotic medication were nonadherent.

In a separate study, adherence was evaluated prospectively using an adherence scale with a range of $1-4$ as follows: 1) the patient had not been prescribed a psychotropic medication, 2) the patient almost always adhered to medication, 3) the patient adhered to the medication half of the time, and 4) the patient almost never adhered to medication. ${ }^{6}$ By using a more narrow definition of nonadherence, the authors found that $\sim 24 \%$ of patients were nonadherent. They also found higher total medical expenditures for the nonadherent patients compared to those who adhered to the prescribed regimen.

There are many causes of nonadherence, including poor insight into the need for medication and concern about adverse effects. ${ }^{4,8}$ Risk factors that increase the likelihood of nonadherence include younger age, substance misuse, homelessness, non-Caucasian ethnicity, being unmarried, and living alone. ${ }^{7,9}$

As would be expected, nonadherence worsens the natural course of the illness. Persons with bipolar disorder who are nonadherent are more likely to miss work, have a reduced work schedule, and have more frequent emergency department visits. ${ }^{9}$ Nonadherence is a major risk factor for relapse, hospital readmission, and suicidality. ${ }^{9}$

Nonadherence is also costly. Inpatient treatment for mental disorders costs $\sim \$ 6,000$ per admission, with an average length of stay of 8 days. ${ }^{10}$ Overall, annual medical expenditures for bipolar disorder are between $\$ 8.5$ billion and $\$ 27.5$ billion. ${ }^{11}$ On an individual basis, the average lifetime cost is $\sim \$ 252,000$ ranging from $\$ 11,720$ for patients with a single manic episode to $\$ 625,000$ in chronic patients and those who do not improve with treatment, often a sign of nonadherence. ${ }^{12}$ A European study found a twofold increase in total costs for inpatient treatment among nonadherent patients when compared to those who were adherent with treatment. ${ }^{6}$ These additional costs can put significant stress on the health care system because patients with mental or substance-use disorders are twice as likely to be uninsured compared to patients without these diagnoses. ${ }^{10}$

Despite the high cost of relapse in both financial and human terms, pharmacologic options for maintaining stability are limited. Lithium continues to be the gold standard treatment for bipolar disorder, especially during the maintenance phase; ${ }^{13}$ however, relapse continues to be the rule. A systematic review found an average risk of relapse rate of $40 \%$ among bipolar patients taking lithium. ${ }^{14}$ While this rate was significantly better than the $60 \%$ relapse rate associated with placebo, it is still far from ideal.

Long-acting injectable (LAI) medication is an attractive option given the high rates of nonadherence among bipolar patients. The first LAI medication approved for the treatment of bipolar disorder was risperidone, and more recently a formulation of LAI aripiprazole has received approval by the Federal Drug Administration for this indication. This article will review the evidence that supports the use of LAI aripiprazole for the treatment of bipolar disorder.

\section{Materials and methods}

We conducted a literature search using the online engines of Google Scholar, PubMed, and Medline. We searched for studies using aripiprazole LAI for the treatment of bipolar disorder. Keywords used included "bipolar," "aripiprazole," "long-acting," "injectable," "LAI," and "long-acting aripiprazole." One clinical trial was identified that focused on the use of LAI aripiprazole in bipolar disorder. Representative articles were selected for illustrative purposes that more broadly discussed the use of aripiprazole in bipolar disorder and LAI aripiprazole for the treatment of psychiatric conditions. 


\section{Results \\ Mechanism of action}

Aripiprazole was discovered by scientists at Otsuka Pharmaceuticals and later licensed to Bristol-Myers Squibb for joint development. ${ }^{15}$ The US Food and Drug Administration (FDA) approved aripiprazole for the treatment of schizophrenia in November 2002. Currently approved indications include the treatment of schizophrenia, acute manic and mixed episodes associated with bipolar I disorder, maintenance treatment of bipolar I disorder, adjunctive treatment of major depressive disorder, irritability associated with autism, Tourette's disorder, and agitation associated with schizophrenia and bipolar mania. ${ }^{16} \mathrm{LAI}$ aripiprazole was approved for the treatment of schizophrenia in 2013. ${ }^{17}$

Aripiprazole interacts with multiple receptors in the brain, which may contribute to its specific clinical effects. It has high affinity for dopamine D2 and D3 receptors and serotonin 5HT1A and 5HT2A receptors, and moderate affinity for dopamine D4 and serotonin 5HT2C and 5HT7 receptors. ${ }^{18}$ Each of these receptor interactions will be briefly reviewed.

Aripiprazole was the first antipsychotic to use a partial agonist strategy to modify the activity of the dopamine system. Prior to its introduction, all antipsychotics blocked the $\mathrm{D} 2$ receptor as antagonists, preventing dopamine, the natural agonist, from binding and bringing about a conformational change that activated the receptor.

Unlike antagonists, partial agonists trigger a conformational change in the receptors they bind to, but the resulting morphology of the receptor is different from the result of a full agonist and leads to a lower level of activation. ${ }^{19}$ This intermediate effect allows aripiprazole to have some degree of functional selectivity, meaning it can increase or decrease $\mathrm{D} 2$ receptor activity depending on its environment. ${ }^{20}$ For example, if there is dopamine overactivity, the partial agonist will displace the full agonist and reduce the response. If, on the other hand, there is a deficiency of dopaminergic activity, the partial agonist will partly restore normal receptor functioning. ${ }^{21}$

Apart from its effects on psychosis, partial D2 agonism may account for other effects of aripiprazole. In the tuberoinfundibular pathway, dopamine acts to suppress the release of prolactin from the pituitary. A troublesome side effect of antipsychotic medication is hyperprolactinemia that occurs when dopamine is prevented from acting in this part of the brain. Aripiprazole, on the other hand, tends to lower prolactin levels, and can be used to counteract hyperprolactinemia caused by other antipsychotic medications. ${ }^{21}$ In terms of side effects, agitation, anxiety, nausea, and insomnia may be due to partial agonism at the D2 receptor. ${ }^{20}$
The dopamine D3 receptor, for which aripiprazole has high affinity as a partial agonist, has been implicated in the pathophysiology of schizophrenia, drug addiction, and mood disorders. ${ }^{22}$ Dopamine D3 receptors are widely distributed throughout the brain with high levels found in the limbic system. ${ }^{22}$ Preclinical studies using cariprazine, another atypical antipsychotic with high affinity to D3 receptors, have found procognitive and mood-altering effects in rats..$^{23,24}$ New D3 antagonists are being developed to treat addiction and compulsive behavior disorders. ${ }^{23}$

Serotonin activity is also modulated by aripiprazole. Aripiprazole acts as a partial agonist at the $5 \mathrm{HT} 1 \mathrm{~A}$ receptor, which mediates inhibitory neurotransmission and is involved in neurogenesis. ${ }^{25,26}$ Dense concentrations of 5HT1A receptors are found in limbic areas, such as the hippocampus, as well as in the cortex, the midbrain and the raphe nuclei. ${ }^{27}$ Systemic administration of $5 \mathrm{HT} 1 \mathrm{~A}$ receptor agonists selectively stimulates 5HT1A receptors located on gamma-aminobutyric (GABA) neurons in the prefrontal cortex. ${ }^{28}$ This effect reduces the activity of GABA neurons, disinhibiting excitatory glutamate neurons, which ultimately results in the activation of mesocortical dopamine neurons and increased activity in the frontal cortex. ${ }^{28}$ This mechanism suggests that 5HT1A receptor agonism may improve cognition in schizophrenia. ${ }^{28}$ As with buspirone, which has a similar mechanism of action, modulation of 5HT1A receptors also may reduce anxiety. ${ }^{29}$

Another way in which aripiprazole may increase dopaminergic neurotransmission is via antagonism of serotonin 5HT2A receptors. ${ }^{21,28}$ The clinical ramifications may include effects on cognition and negative symptoms.

Aripiprazole is a weak partial agonist at the serotonin 5HT7 receptor. Dense concentrations of this receptor have been found in the hypothalamus, thalamus, hippocampus, brainstem, and cortex. ${ }^{30}$ Consistent with this anatomical distribution, evidence derived from animal studies have linked 5HT7 receptors to thermoregulation, learning, memory, endocrine function, circadian rhythm, and sleep. ${ }^{27,30}$ Drugs that stimulate 5HT7 receptors have been shown to induce circadian phase shifts. ${ }^{27,31}$ In addition, selective 5HT7 antagonists given to animals increased the latency to rapid eye movement (REM) sleep and decreased the amount of time spent in REM sleep - the opposite of what is encountered in depressed patients. ${ }^{31}$

Activity at the serotonin $5 \mathrm{HT} 2 \mathrm{C}$ receptor appears to have effects on feeding behavior, especially in conjunction with histamine $\mathrm{H} 1$ receptor antagonism. ${ }^{21}$ Aripiprazole acts as a partial agonist at 5HT2C. Per Disciasio, the antagonist component of aripiprazole's partial agonist activity at the 
serotonin 5HT2C receptor may be responsible for weight gain. The explanation for this effect is based on the finding that activation of $5 \mathrm{HT} 2 \mathrm{C}$ results in increased satiety and hypophagia in animal studies. ${ }^{32}$

Aripiprazole has varying effects on weight. ${ }^{33}$ Studies that enrolled participants with schizophrenia did not find significant weight gain associated with aripiprazole exposure. However, when aripiprazole was used to augment serotonergic antidepressants for the treatment of refractory depression, weight gain occurred significantly more frequently than with placebo. $^{34}$

As previously noted, aripiprazole is a partial agonist at 5HT2C. Nguyen et al hypothesize that aripiprazole may act as an antagonist when exposed to high serotonergic activity, potentially increasing the risk for weight gain. In low serotonergic environments, it may act as an agonist and have the reverse effect. In this context, the presence of the serotonergic antidepressant would explain the weight gain seen in the depression studies as opposed to the weight-neutral effect seen in the schizophrenia studies. To test this hypothesis, they conducted a retrospective chart review of patients prescribed aripiprazole with high serotonergic antidepressants (citalopram, paroxetine, sertraline, or venlafaxine) versus bupropion, which has minimal effect on serotoninergic activity. They noted a statistically significant mean increase in weight among patients taking aripiprazole with high serotonergic antidepressant. In the aripiprazole plus bupropion group, they noted a decrease in weight, although it was not clinically significant.

\section{Long-acting injectable antipsychotics}

To produce LAI forms of the older, typical antipsychotics, such as haloperidol and fluphenazine, a long-chain fatty acid moiety, such as decanoate or enanthate, is added to the drug, so it can be dissolved in sesame oil, which is used as the delivery vehicle. The drug is absorbed from the surface of the depot injection, and in the case of haloperidol, no overlap with oral medication is required when a loading dose strategy is used. ${ }^{35}$

Atypical LAI antipsychotics use water as the vehicle for delivery. Risperidone, the first atypical LAI, is delivered in the form of a biodegradable microsphere preparation. ${ }^{35,36}$ It has a similar side-effect profile as oral risperidone, and the main challenges in the clinical setting have been the need for refrigeration as well as the need to dose the medication every 2 weeks. ${ }^{36}$ A period of overlap with oral medication is required with LAI risperidone.

LAI olanzapine uses a pamoate moiety suspended in water and does not require oral supplementation overlap. ${ }^{35}$
The greatest clinical challenge has been postinjection delirium syndrome, characterized by sudden sedation, confusion, slurred speech, altered gait, or unconsciousness following delivery. Patients must be observed for 3 hours after receiving an injection. ${ }^{35,36}$

Paliperidone is the active metabolite of risperidone and is also available as an LAI preparation. It has a similar adverse effect profile. Unlike risperidone, there is no need for oral supplementation during the initial treatment phase. An even greater advantage is that it can be given monthly, and there is no need for refrigeration, which makes it easier to handle. ${ }^{35,36}$

LAI aripiprazole was approved for the treatment of schizophrenia in 2013. ${ }^{17}$ The contraindications, warnings, precautions, and side effects of long-acting ones are similar to the oral formulation. Aripiprazole LAI is available as a lyophilized powder, which needs to be reconstituted with sterile water prior to administration. ${ }^{17,35,36}$ Release into the bloodstream is slow, and it requires 14 days of overlap with oral aripiprazole to maintain a therapeutic concentration. $^{37}$

With LAI antipsychotics, the most important factor that influences the pharmacokinetic behavior is absorption from the injection site. ${ }^{36}$ With aripiprazole, the absorption rate is slower than the elimination rate. ${ }^{38}$ This phenomenon, in which the drug disposition is limited by absorption rather than metabolism, is called "flip-flop kinetics." 36 The half-life is dependent on release rather than elimination. ${ }^{39}$ Slow absorption is primarily due to the poor solubility of the particles. ${ }^{36}$

Both aripiprazole and its major metabolite, dehydroaripiprazole, bind to albumin, and at therapeutic levels, they are $99 \%$ bound. Aripiprazole is metabolized by the P450 system, mainly by isoenzymes 3A4 and 2D6. Dosing adjustments are recommended when it is used with inhibitors of CYP3A4 (eg, ketoconazole) or inhibitors of CYP2D6 (eg, quinidine, fluoxetine, and paroxetine). Reducing the dose by one-half is appropriate when coadministering it with these agents. ${ }^{20}$ Carbamazepine, via induction of CYP3A4, may reduce aripiprazole levels by as much as $70 \%{ }^{40}$ Consequently, aripiprazole should generally not be used in combination with inducers of CYP3A4 $4{ }^{41}$ Caution is recommended when aripiprazole is used with antihypertensive agents because of its alpha adrenergic antagonism and potential to enhance the effects of these agents. ${ }^{41}$ If this combination is used, patients should be educated on the signs and symptoms of hypotension, and blood pressure should be carefully monitored. Use in combination with benzodiazepines also carries the risk of hypotension as well as excess sedation. ${ }^{41}$ 
No dosing adjustment is needed when aripiprazole is used with valproate, and there are no significant reports of any other interactions with P450 enzymes. ${ }^{41}$ Approximately $8 \%$ of Caucasians and 3\% of African Americans are poor metabolizers of CYP 2D6 substrates. In the clinical setting, anyone with unusual side effects at low doses should be considered a poor metabolizer. ${ }^{17}$ No dosing adjustments are required based on sex, age, race, smoking status, and renal or hepatic impairment. ${ }^{41}$

\section{The efficacy of aripiprazole in the treatment of bipolar disorder}

There are several studies examining the safety and efficacy of oral aripiprazole for the treatment of acute manic episodes of bipolar disorder. One large-scale prospective trial enrolled 272 hospitalized patients in a double-blind, placebocontrolled, 3-week study. The primary outcome measure was mean change in the Young Mania Rating Scale (YMRS). Aripiprazole demonstrated superior efficacy to placebo in the treatment of patients with bipolar I disorder presenting with acute manic or mixed episodes. ${ }^{42}$ The discontinuation rates due to adverse events were similar for aripiprazole and placebo, and $80 \%$ of aripiprazole-treated patients maintained the $30 \mathrm{mg}$ /day target dose. A 2011 meta-analysis on the use of aripiprazole for acute mania included a total of 2,333 patients who took part in a randomized controlled trial. ${ }^{43}$ The number needed to treat was 6 for response and 14 for remission at week 3 of the study. On average, the response to aripiprazole began at day 3 , and suicide rates were found to be negligible in all groups with mania.

Based on a post hoc analysis, aripiprazole appears to be effective in people with rapid cycling bipolar disorder. Twenty-eight patients with the rapid cycling subtype who were treated with aripiprazole for a mixed or manic episode, and then followed for 100 weeks experienced outcomes that were similar to the non-rapid cycling participants. ${ }^{44}$

The efficacy of aripiprazole for the treatment of elevated mood does not seem to be affected by the presence of mixed symptoms. A post hoc analysis of a randomized, placebocontrolled trial that included 516 participants with acute manic or mixed episodes found that aripiprazole was effective regardless of baseline depressive symptoms, which were measured using the Montgomery Asberg Depression Rating Scale (MADRS). ${ }^{45}$ Furthermore, aripiprazole significantly reduced YMRS scores at the 3-week endpoint compared with placebo in patients with more severe or less severe illness, with or without psychotic features, and with or without a history of rapid cycling. There was no significant difference in response observed in men or women. Thus, aripiprazole was found to be helpful across a range of subpopulations experiencing acutely manic or mixed episodes.

Aripiprazole has not been found to be effective for the treatment of bipolar depression. A 2008 multicenter, randomized, placebo-controlled study evaluated the efficacy and safety of aripiprazole monotherapy in patients experiencing a bipolar depressive episode without psychotic features. The primary end point was mean change from baseline to week 8 in the MADRS score. In this study, aripiprazole did not achieve statistical significance versus placebo at week $8 .{ }^{46}$ Additional studies have reported similar results, indicating that aripiprazole is not effective for treatment of acute bipolar depressive episodes. ${ }^{43}$

In addition to its effectiveness for acute manic and mixed episodes, aripiprazole oral formulation has also been found to be effective in the maintenance treatment of bipolar disorder. The drug was granted approval for this indication by the FDA in 2005. The pivotal study examining efficacy and tolerability of aripiprazole for relapse prevention in bipolar I disorder followed patients for 100 weeks. ${ }^{47}$ This longitudinal study featured 26 weeks of double-blind treatment with either aripiprazole or placebo, with a primary endpoint of time to relapse for any mood episode. At 100 weeks, time to relapse was significantly longer with aripiprazole than with placebo, specifically delaying time to manic relapse. There was no significant difference between placebo and aripiprazole in time to depressive relapse.

In addition to this longitudinal study, a 2011 metaanalysis also examined the efficacy of oral aripiprazole for maintenance prevention of manic episodes in a population who had been responsive to the medication for treatment of acute decompensation. ${ }^{43}$ Researchers found the median survival time (measured as time to any mood episode) for the aripiprazole group to be longer than the study could measure, while the median survival of the placebo group was 118-203 days. ${ }^{43}$

\section{LAl aripiprazole in schizophrenia}

The first indication for LAI aripiprazole was for the treatment of schizophrenia. A large study that enrolled 710 participants examined the efficacy and tolerability of the LAI formulation in the maintenance phase of schizophrenia. Participants entered the study during acute exacerbations, and were first treated with open-label oral aripiprazole. Five hundred seventy-six participants were able to receive LAI, and of these, 403 were adequately stabilized and able to enter the randomized, placebo-controlled phase of the study. LAI aripiprazole was found to significantly delay time to relapse in comparison with placebo. ${ }^{48}$ In another study, once-monthly 
$400 \mathrm{mg}$ LAI aripiprazole was shown to be noninferior to the oral formulation. ${ }^{49}$

\section{LAI aripiprazole in bipolar disorder}

In July 2017, the FDA approved LAI aripiprazole for maintenance monotherapy of bipolar I disorder in adults. This approval was based on a single study that included over 700 individuals. It utilized a diverse sample of participants recruited from 103 sites in 7 countries. ${ }^{50}$ The outcome was highly significant - both statistically and clinically. Nearly twice as many LAI-treated participants remained stable for the entire study compared to those treated with placebo.

As with any study, there are aspects that require the clinician to use caution when applying the results to patient care. An efficacy trial, in which the goal is to evaluate whether a drug works better than placebo, is designed to maximize internal validity, that is, the likelihood that the difference between an effective drug and placebo will achieve statistical significance. This design is an essential first step in the evaluation of a medication, but maximizing internal validity can reduce the generalizability of the findings. One component of this trade-off is subject selection.

Similar to the study of LAI in schizophrenia, this study used an enriched study sample, enrolling participants who had a greater than average chance of remaining stable on LAI. Participants were selected based on their ability to transition, one step at a time, from the medications they were taking at study entry to monotherapy with oral aripiprazole and then to LAI aripiprazole. Participants who became unstable at any point along the way were removed from the study prior to randomization.

The use of an enriched sample is standard practice in bipolar disorder maintenance studies. Although it necessarily limits the generalizability of the findings, it matches the way medications are used in clinical practice. For example, if a potential maintenance drug is used for acute stabilization and fails, clinicians are unlikely to continue it into the maintenance phase of treatment.

In clinical trials, the specific inclusion criteria that are used to enrich the sample may be more or less restrictive. In this study, the first step was to ensure that the participants who were taking other medications for bipolar disorder could successfully transition to oral aripiprazole. Of the 731 people who were eligible for the study, 466 needed to be transitioned (the others were either on aripiprazole already or on nothing because of nonadherence). This phase of the study lasted 4-6 weeks, and resulted in about a quarter of the participants being removed from the study.
The next phase was to select individuals who could be stabilized on oral aripiprazole monotherapy. This phase lasted 2-8 weeks, and caused about a third of the remaining sample to be excluded.

After stabilization on oral aripiprazole, the third phase required participants to transition to $\mathrm{LAI}$, and maintain stability on LAI monotherapy for at least 8 consecutive weeks. Participants were given $400 \mathrm{mg}$ of aripiprazole at each monthly injection with the option to decrease the dose to $300 \mathrm{mg}$ if tolerability became a problem. Approximately $65 \%$ of those stabilized on the oral formulation remained stable on LAI.

In all, 465 participants were removed from the study because they did not do well while being transitioned from their baseline treatment to LAI. The remaining 266 participants were eligible for randomization to either LAI continuation at 300 or $400 \mathrm{mg}$ per month (mean dose $380 \mathrm{mg}$ ) or a switch to placebo.

Although LAI aripiprazole was substantially more effective than placebo, only about half of the participants who made it to the randomization phase and were given LAI aripiprazole remained stable for the entire 52-week study period. Among those who were randomized to placebo, a little more than a quarter remained stable. These numbers reflect the fact that bipolar disorder is a cyclic illness, and it is unrealistic to expect maintenance treatment to eliminate abnormal mood episodes completely. These results also highlight the challenges of treating bipolar disorder with monotherapy. In clinical practice, fewer than $20 \%$ of bipolar patients are treated with mood stabilizer monotherapy. Most require at least three medications. ${ }^{51}$ Further emphasizing the need for combined therapy is LAI aripiprazole's relative lack of efficacy preventing depressive episodes.

Treatment-emergent adverse events were, for the most part, mild to moderate. Akathisia was the most common adverse event, which, combined with restlessness, was experienced by $23 \%$ of the sample. Approximately $11 \%$ of participants experienced weight gain, 10\% insomnia, 7\% anxiety, and $5 \%$ fatigue.

\section{Conclusion}

Although the data supporting LAI aripiprazole as an effective treatment for the maintenance phase of bipolar disorder are currently limited to a single study, the results were robust enough to merit FDA approval for this indication. This is a welcome development. Despite the numerous medications that are available to treat this disorder, none is fully effective for all patients and the problem of long-term adherence 
remains a significant challenge. Future studies will be needed to confirm the disappointing finding that LAI aripiprazole does not seem to be more effective than placebo for the prevention of new depressive episodes. Additionally, combination treatment with lithium, anticonvulsant medications, and other mood stabilizers should be investigated to determine whether efficacy can be further improved. Combination with lamotrigine would be of particular interest, given that medication's unique efficacy in preventing depressive episodes during maintenance.

In some ways, the maintenance phase is the most important phase of treatment. Acute manic episodes have the most dramatic presentations, and acute depressive episodes, along with long-term residual depressive symptoms, account for the majority of disability experienced by people living with bipolar disorder. ${ }^{52}$ However, it is during the maintenance phase, when patients are relatively symptom-free, that they live their lives. It is when they pursue their careers, develop relationships, and discover their identity as a unique individual, separate from the personality-distorting symptoms of the illness. Psychiatrists tend to focus on pathology; however, only wellness can lead to recovery. Despite the lack of symptomatology, maintenance is an aspect of the bipolar disorder that deserves a high priority.

\section{Disclosure}

The authors report no conflicts of interest in this work.

\section{References}

1. Merikangas KR, Jin R, He JP, et al. Prevalence and correlates of bipolar spectrum disorder in the world mental health survey initiative. Arch Gen Psychiatry. 2011;68(3):241-251.

2. Ferri F, Raus V. Bipolar Disorder. In: Ferri's Clinical Advisor 2018. Philadelphia, USA: Elsevier; 2018:190-191.

3. Velligan DI, Lam YW, Glahn DC, et al. Defining and assessing adherence to oral antipsychotics: a review of the literature. Schizophr Bull. 2006;32(4):724-742.

4. Mitchell AJ, Selmes T. Why don't patients take their medicine? Reasons and solutions in psychiatry. Adv Psychiatr Treat. 2007;13(5): 336-346.

5. Colom F, Vieta E, Tacchi MJ, Sanchez-Moreno J, Scott J. Identifying and improving non-adherence in bipolar disorders. Bipolar Disord. 2005; 7(Suppl 5):24-31.

6. Hong J, Reed C, Novick D, Haro JM, Aguado J. Clinical and economic consequences of medication non-adherence in the treatment of patients with a manic/mixed episode of bipolar disorder: results from the European Mania in Bipolar Longitudinal Evaluation of Medication (EMBLEM) Study. Psychiatry Res. 2011;190(1):110-114.

7. Sajatovic M, Valenstein M, Blow FC, Ganoczy D, Ignacio RV. Treatment adherence with antipsychotic medications in bipolar disorder. Bipolar Disord. 2006;8(3):232-241.

8. Novick D, Montgomery W, Treuer T, Aguado J, Kraemer S, Haro JM. Relationship of insight with medication adherence and the impact on outcomes in patients with schizophrenia and bipolar disorder: results from a 1-year European outpatient observational study. BMC Psychiatry. 2015;15(1):189.
9. Rascati KL, Richards KM, Ott CA, et al. Adherence, persistence of use, and costs associated with second-generation antipsychotics for bipolar disorder. Psychiatr Serv. 2011;62(9):1032-1040.

10. Heslin KC, Elixhauser A, Steiner CA. Hospitalizations involving mental and substance use disorders among adults, 2012: Statistical Brief \#191. HCUP Stat Br. 2015.

11. Kleinman L, Lowin A, Flood E, Gandhi G, Edgell E, Revicki D. Costs of bipolar disorder. Pharmacoeconomics. 2003;21(9):601-622.

12. Begley CE, Annegers JF, Swann AC, et al. The lifetime cost of bipolar disorder in the US: an estimate for new cases in 1998. Pharmacoeconomics. 2001;19(5 Pt 1):483-495.

13. Perlis RH, Ostacher MJ. Lithium and Its Role in Psychiatry. In: Stern TS, Fava M, Wilens TE, Rosenbaum JF, editors. Massachusetts General Hospital Comprehensive Clinical Psychiatry. 2nd ed. China: Elsevier, Inc. 2016:47:525-531.

14. Geddes JR, Burgess S, Hawton K, Jamison K, Goodwin GM. Longterm lithium therapy for bipolar disorder: systematic review and metaanalysis of randomized controlled trials. Am J Psychiatry. 2004;161(2): 217-222.

15. Bristol Meyers Squibb, Otsuka Holdings (2002). Bristol Meyers Squibb, Otsuka Holdings (2002) Abilify ${ }^{\mathrm{TM}}$ (Aripiprazole) Approved By U.S. Food And Drug Administration For Treatment Of Schizophrenia. [online press release] Available from: http://www.evaluategroup.com/Universal/ View.aspx?type=Story\&id=34150. Accessed December 3, 2017.

16. Abilify ${ }^{\circledR}$ (Aripiprazole) [Prescribing Information]. Rockville, MD: Otsuka America Pharmaceutical, Inc.

17. Texas Department of State Health Services: Aripiprazole for Extended Release Injectable Suspension [Formulary Monograph]; 2013.

18. Abilify $^{\circledR}$ (Aripiprazole) [Package Insert]. Rockville, MD: Otsuka America Pharmaceutical, Inc.

19. Wecker L, Watts S, Faingold C, Dunaway G, Crespo L. Brody's Human Pharmacology-E-Book; Philadelphia, USA: Mosby Inc. 2010.

20. Davies MA, Sheffler DJ, Roth BL. Aripiprazole: a novel atypical antipsychotic drug with a uniquely robust pharmacology. CNS Drug Rev. 2006;10(4):317-336.

21. Sciascio G Di, Riva M. Aripiprazole: from pharmacological profile to clinical use. Neuropsychiatr Dis Treat. 2015;11:2635-2647.

22. Leggio GM, Salomone S, Bucolo C, et al. Dopamine D3 receptor as a new pharmacological target for the treatment of depression. Eur $J$ Pharmacol. 2013;719(1-3):25-33.

23. Pich EM, Collo G. Pharmacological targeting of dopamine D3 receptors: possible clinical applications of selective drugs. Eur Neuropsychopharmacol. 2015;25(9):1437-1447.

24. Papp M, Gruca P, Lason-Tyburkiewicz M, Adham N, Kiss B, Gyertyán I. Attenuation of anhedonia by cariprazine in the chronic mild stress model of depression. Behav Pharmacol. 2014;25(5-6):567-574.

25. Jordan S, Koprivica V, Chen R, Tottori K, Kikuchi T, Altar CA. The antipsychotic aripiprazole is a potent, partial agonist at the human 5-HT1A receptor. Eur J Pharmacol. 2002;441(3):137-140.

26. Mahar I, Bambico FR, Mechawar N, Nobrega JN. Stress, serotonin, and hippocampal neurogenesis in relation to depression and antidepressant effects. Neurosci Biobehav Rev. 2014;38:173-192.

27. Naumenko VS, Popova NK, Lacivita E, Leopoldo M, Ponimaskin EG. Interplay between serotonin 5-HT1A and 5-HT7 receptors in depressive disorders. CNS Neurosci Ther. 2014;20(7):582-590.

28. Kusumi I, Boku S, Takahashi Y. Psychopharmacology of atypical antipsychotic drugs: from the receptor binding profile to neuroprotection and neurogenesis. Psychiatry Clin Neurosci. 2015;69(5): 243-258.

29. Blier $\mathrm{P}$, Ward NM. Is there a role for 5 -HT1A agonists in the treatment of depression? Biol Psychiatry. 2003;53(3):193-203.

30. Mnie-Filali O, Lambas-Senas L, Scarna H, Haddjeri N. Therapeutic potential of 5-HT7 receptors in mood disorders. Curr Drug Targets. 2009; 10(11):1109-1117.

31. Hedlund PB, Sutcliffe JG. Functional, molecular and pharmacological advances in 5-HT 7 receptor research. Trends Pharmacol Sci. 2004; 25(9):481-486. 
32. Bickerdike MJ. 5-HT2C receptor agonists as potential drugs for the treatment of obesity. Curr Top Med Chem. 2003;3(8):885-897.

33. Nguyen CT, Rosen JA, Bota RG. Aripiprazole partial agonism at 5-HT2C: a comparison of weight gain associated with aripiprazole adjunctive to antidepressants with high versus low. Prim Care Companion CNS Disord. 2012;14(5): pii: PCC. 12m01386.

34. Marcus RN, McQuade RD, Carson WH, et al. The efficacy and safety of aripiprazole as adjunctive therapy in major depressive disorder. J Clin Psychopharmacol. 2008;28(2):156-165.

35. Gopalakrishna G, Aggarwal A, Lauriello J. Long-acting injectable aripiprazole: how might it fit in our tool box? Clin Schizophr Relat Psychoses. 2013;7(2):87-92.

36. Meyer JM. Understanding depot antipsychotics: an illustrated guide to kinetics. CNS Spectr. 2013;18(Suppl 1):58-67.

37. Mallikaarjun S, Kane JM, Bricmont P, et al. Pharmacokinetics, tolerability and safety of aripiprazole once-monthly in adult schizophrenia: an open-label, parallel-arm, multiple-dose study. Schizophr Res. 2013;150(1):281-288

38. Johnstone EC. Companion to Psychiatric Studies. 8th ed. China: Churchill Livingstone; 2010.

39. Taylor D. Psychopharmacology and adverse effects of antipsychotic long-acting injections: a review. Br J Psychiatry Suppl. 2009;52: S13-S19.

40. Goodnick PJ, Jerry JM. Aripiprazole: profile on efficacy and safety. Expert Opin Pharmacother. 2002;3(12):1773-1781.

41. Abilify Maintena ${ }^{\circledR}$ (Aripiprazole) [Prescribing Information]. Rockville, MD: Otsuka America Pharmaceutical, Inc; 2017.

42. Sachs G, Sanchez R, Marcus R, et al; Aripiprazole Study Group. Aripiprazole in the treatment of acute manic or mixed episodes in patients with bipolar I disorder: a 3-week placebo-controlled study. J Psychopharmacol. 2006;20(4):536-546.

43. Fountoulakis KN, Vieta E, Schmidt F. Aripiprazole monotherapy in the treatment of bipolar disorder: a meta-analysis. J Affect Disord. 2011; 133(3):361-370
44. Muzina DJ, Momah C, Eudicone JM, et al. Aripiprazole monotherapy in patients with rapid-cycling bipolar I disorder: an analysis from a longterm, double-blind, placebo-controlled study. Int J Clin Pract. 2008; 62(5):679-687.

45. Suppes T, Eudicone J, McQuade R, Pikalov A 3rd, Carlson B. Efficacy and safety of aripiprazole in subpopulations with acute manic or mixed episodes of bipolar I disorder. J Affect Disord. 2008;107(1-3): 145-154.

46. Thase ME, Jonas A, Khan A, et al. Aripiprazole monotherapy in nonpsychotic bipolar I depression: results of 2 randomized, placebo-controlled studies. J Clin Psychopharmacol. 2008;28(1):13-20.

47. Keck PE Jr, Calabrese JR, McIntyre RS, et al; Aripiprazole Study Group. Aripiprazole monotherapy for maintenance therapy in bipolar I disorder: a 100-week, double-blind study versus placebo. J Clin Psychiatry. 2007;68(10):1480-1491.

48. Kane JM, Sanchez R, Perry PP, et al. Aripiprazole intramuscular depot as maintenance treatment in patients with schizophrenia: a 52-week, multicenter, randomized, double-blind, placebo-controlled study. J Clin Psychiatry. 2012;73(5):617-624.

49. Fleischhacker WW, Baker RA, Eramo A. Effects of aripiprazole once-monthly on domains of personal and social performance: results from 2 multicenter, randomized, double-blind studies. Schizophr Res. 2014; 159(2-3):415-420.

50. Calabrese JR, Sanchez R, Jin N, et al. Efficacy and safety of aripiprazole once-monthly in the maintenance treatment of bipolar I disorder: a double-blind, placebo-controlled, 52-week randomized withdrawal study. J Clin Psychiatry. 2017;78(3):324-331.

51. Ghaemi SN, Hsu DJ, Thase ME, et al. Pharmacological treatment patterns at study entry for the first 500 STEP-BD participants. Psychiatr Serv. 2006;57(5):660-665.

52. Judd LL, Akiskal HS, Schettler PJ, et al. The long-term natural history of the weekly symptomatic status of bipolar I disorder. Arch Gen Psychiatry. 2002;59(6):530-537.
Neuropsychiatric Disease and Treatment

\section{Publish your work in this journal}

Neuropsychiatric Disease and Treatment is an international, peerreviewed journal of clinical therapeutics and pharmacology focusing on concise rapid reporting of clinical or pre-clinical studies on a range of neuropsychiatric and neurological disorders. This journal is indexed on PubMed Central, the 'PsycINFO' database and CAS,

\section{Dovepress}

and is the official journal of The International Neuropsychiatric Association (INA). The manuscript management system is completely online and includes a very quick and fair peer-review system, which is all easy to use. Visit http://www.dovepress.com/testimonials.php to read real quotes from published authors. 